\title{
Formula-fed and Clostridioides difficile infected neonatal piglets carry a highly diverse and abundant set of antimicrobial resistance genes
}

Robert Pieper ( $\nabla$ robert.pieper@fu-berlin.de )

Freie Universitat Berlin https://orcid.org/0000-0002-0292-1482

\section{Temesgen Dadi}

Freie Universität Berlin

Lukasz Grzeskowiak

Freie Universität Berlin

\section{Laura Pieper}

Freie Universität Berlin

\section{Britta Siegmund}

Charite Universitatsmedizin Berlin

\section{Wilfried Vahjen}

Freie Universität Berlin

\section{Stefan Schwarz}

Freie Universität Berlin

\section{Femke Heinsen}

Christian-Albrechts-Universitat zu Kiel

\section{Andre Franke}

Christian-Albrechts-Universitat zu Kiel

\section{Knut Reinert}

Freie Universität Berlin

Jürgen Zentek

Freie Univesität Berlin

\section{Research Article}

Keywords: Neonates, Piglets, Formula, Clostridioides difficile, antibiotic resistance, microbiome, resistome

Posted Date: August 13th, 2020

DOI: https://doi.org/10.21203/rs.3.rs-57299/v1 
License: (c) (i) This work is licensed under a Creative Commons Attribution 4.0 International License. Read Full License 


\section{Abstract}

Background Clostridioides difficile infection (CDI) is an increasing zoonotic health threat and has also been documented as a cause of enteritis outbreaks in neonatal pigs. Furthermore, CDI in neonatal piglets cause changes in microbial gut colonization. We hypothesized that an imbalanced microbial colonization in piglets with CDI could be associated with an altered abundance of antimicrobial resistance genes.

Results We analyzed fecal metagenomic data of lactating sows (S), their piglets during suckling (SP), the same piglets two weeks after weaning (WP), 5-day old artificially reared and formula-fed siblings (FP) and FP infected with $C$. difficile (FP-CD) for microbiota composition and antimicrobial resistance gene abundance. FP and FP-CD piglets had an immature-type microbiota and increased abundance of antimicrobial resistance genes. A co-occurrence of genes encoding for resistance against aminoglycosides (e.g. $a p h\left(3^{\prime \prime}\right)-I b$, aph(6)-Id, ant(2")-la), $\beta$-lactams (bla ${ }_{C T X-M^{\prime}}$ bla $\left.a_{T E M}\right)$, fluoroquinolones (pat(A) macrolides ( $m p h(\mathrm{~A})$ ), sulfonamides (sul1, sul2), polypeptides (e.g. pmrB, pmrC, arnA, bac(A)) and tetracyclines (e.g. tet(A-D),) was observed. A strong association between Escherichia, Streptococcus, Shigella, Enterococcus and Fusobacterium taxa with the expansion of the resistome in FP and FP-CD piglets were found.

Conclusion Increased abundance of antimicrobial resistance genes in formula feeding and concomitant CDI may be associated with therapeutic resistance later in life and warrant further studies.

\section{Background}

Bacterial resistance to antibiotics is one of the main public health issues worldwide [1]. The use of antimicrobial agents in clinical practice, veterinary medicine, agriculture and aquaculture has increased the appearance and spread of multi-resistant bacteria. However, there is a need to identify other factors that may facilitate the expansion of antimicrobial resistance (AR) and their possible distribution into the environment. The gastrointestinal tract of humans and pigs is a densely populated habitat and is considered a "hotspot" for the development and hidden selection of AR and/or multidrug resistance genes called the gut resistome [2, 3]. Indeed, AR genes are ubiquitously present among the porcine gut microbiota. High abundance of AR in the absence of antibiotic selective pressure and the variability between pigs under the same rearing conditions has recently been described [4]. However, the overuse of antibiotics is known to put selection pressure on AR, which may contribute to a development of multiresistance among microorganisms. It is well established in pigs that antibiotic growth promoters or therapeutically administered antibiotics will affect both, the intestinal microbiome and the abundance of AR genes [5]. However, bacterial infections and dysbioses may also promote the re-assortment of plasmid-encoded genes (including AR genes) within these microbial communities [6].

In humans, Clostridioides difficile (CD) is a leading cause of nosocomial and community acquired infection (CD infection, $\mathrm{CDI}$ ), morbidity and fatality. In addition, clinical isolates of CD often show multiresistance [7], which may increase the pool and dissemination of AR genes in the gut environment. 
Interestingly, shifts in the intestinal microbiome were recently linked with resistome changes in patients suffering from recurrent CDI [8]. In pigs, spontaneous outbreaks of CDI have been increasingly observed in neonatal suckling piglets at $<14$ days of age [9]. Moreover, hypervirulent ribotypes can be found in pigs and human patients alike [10]. Typing studies revealed high genetic similarities between veterinary and clinical isolates, which suggests that $C D$ may possess both zoonotic and anthroponotic threads [11]. Although the predisposing factors for CDI in neonatal pigs are largely not known, diet may play an important role [12]. Because hyperprolifacy in modern pig breeds has increased the average numbers of life-born piglets per litter (often exceeding the nursing capacity of the mother), artificial fostering systems with formula feeding are more and more used in the pig industry, which may increase the prevalence of CDI. However, little is yet known about the consequences of formula feeding and CDI on the intestinal resistome in neonatal piglets.

\section{Results}

In order to generate intestinal microbiome data of genetically closely related individuals (full- and half-sib piglets), purebred sows ('German Landrace') and their suckling and weaned piglets were used in the study. Fecal samples were taken from four sows (S) and a pooled sample from their suckling piglets $(\mathrm{n}=$ 4) at day 5 (suckling piglets; SP) and two weeks after the weaning at day 42 ( $n=8$ weaned piglets, WP). Fecal samples from FP $(n=3)$ and FP-CD $(n=3)$ animals were taken on day 5 . Using a novel Species Level Identification of Microorganisms from Metagenomes (SLIMM) tool, a total of 1070 bacterial species within 134 genera and 7 major phyla were identified. Lactobacillus, Clostridium and Turicibacter were predominant in S, whereas Bacteroides, Alistipes, Butyricimonas, Desulfovibrio and Lachnoclostridium dominated in SP (Fig. 1B). In WP, Lactobacillus, Bifidobacterium, Roseburia, Megasphaera, Methanobrevibacter, Mitsoukella and Prevotella were the predominant genera, whereas Escherichia, Streptococcus, Shigella, Enterococcus, Collinsella, Fusobacterium and Ruminococcus showed a high abundance in FP-CD and FP piglets. Higher levels of CD were also detected in FP pigs (Fig. 1C).

A total of 83 AR genes were identified within 11 antibiotic classes. The AR gene abundance was lower in S, SP, FP and WP groups as compared to FP and FP-CD piglets (Fig. 2A). Relative abundance of AR genes revealed that all animal groups clustered according to AR gene abundance (Fig. 2B). To determine the association of the 35 most abundant AR genes with the abundance with bacterial genera in the groups, we employed a redundancy analysis (Fig. 2C). The abundance of AR genes related to tetracycline (tet(Q), tet(W)), lincosamide (Inu(C), Isa(E)), and aminoglycoside resistance (ant(6)-Ib) in S, SP and WP metagenomes coincided with the abundance of the bacterial genera such as Megasphaera, Bifidobacterium, Lactobacillus, Clostridium, and Prevotella. RDA analysis further confirmed the strong association between the genera Escherichia, Streptococcus, Shigella, Enterococcus and Fusobacterium with the expansion of the resistome in FP and FP-CD piglets. Co-occurrence of genes encoding for resistance against aminoglycosides (e.g. aph(3")-Ib, aph(6)-ld, ant(2")-la), $\beta$-lactams (b/a ${ }_{C T X-{ }_{M}}$ bla $\left.{ }_{T E M}\right)$, 
fluoroquinolones (pat(A) macrolides (mph(A)), sulfonamides (sul1), polypeptides (e.g. pmrB, pmrC, arnA, $\operatorname{bac}(\mathrm{A}))$ and tetracyclines (e.g. $\operatorname{tet}(\mathrm{B})$, $\operatorname{tet}(\mathrm{D}))$ was observed.

\section{Discussion}

The complex intestinal microbial ecosystem is characterized by a close microbe-microbe-host relationship, which reaches a climax status. However, this homeostasis can be shifted into a state of dysbiosis by e.g. antimicrobial agents, diet, immune deficiency or infection, and thereby possibly facilitate the expansion of the resistome [6]. Despite the well-studied and highly diverse microbial ecosystem in older individuals, the colonization of the neonatal gut in both humans and pigs is a de novo establishment of a microbial ecosystem undergoing several consecutive steps under the influence of genetic, maternal, and environmental (dietary and medical) factors $[13,14,15]$. Moreover, the early neonatal phase seems to determine the microbial profile and intestinal health later in life. In pigs, the very early colonizers between birth and 2 days of age are mainly enterobacteria, streptococci and clostridia of maternal and environmental origin $[13,14,16]$. This is supported by our findings here, where Escherichia, Streptococcus, Shigella, Enterococcus, Collinsella, Fusobacterium and Ruminococcus were the dominating genera in FP and FP-CD piglets. Under normal conditions, these genera become later outcompeted by others such as Alistipes, Bacteroides, Fusobacterium and Lactobacillus $[13,16]$. Because FP and FP-CD piglets were transferred into the artificial rearing units within $6 \mathrm{~h}$ after birth and had no further contact to the mothers surroundings, it seems likely that these piglets habored an 'immature-like' microbiome as compared with SP and WP piglets, accompanied by a further expansion of these early colonizers through the formula diet. For example, formula with high lactose content promoted the proliferation of enterobacteria in neonatal piglets [17]. We have previously shown that formula feeding also promotes $\mathrm{CDI}$ in neonatal piglets and the expansion of naturally colonizing $\mathrm{CD}$ in FP piglets [12]. This is in line with studies in formula-fed infants showing a higher intestinal colonization with $C D$ and $E$. coli as compared to breast-fed infants $[18,19]$. Breastmilk is considered the gold standard for infant nutrition and this same is true for neonatal piglets. It seems likely that maternal antibodies in the porcine milk may provide protection against CDI in the suckling offspring. For example, we have recently shown that sow colostrum and serum contain antibodies against CD toxin A [20]. In addition, sow colostrum exerted a protective effect against CD toxin-induced effects in porcine intestinal cell model IPEC-J2 [21]. A formula-induced altered or delayed intestinal colonization and low abundance of lactobacilli or clostridia may decrease colonization resistance thereby promoting $C D$ dissemination $[16,22,23]$. The expansion of Escherichia, Streptococcus, Shigella, Enterococcus phylotypes through the formula diet is of particular relevance because they represent a presumed source of persisting multi-drug resistant pathogens through further forward selection under medical treatment or other stress factors in the gut environment later in life. Interestingly, strong positive correlations between CD counts and abundance of Staphylococcus spp., Enterococcus spp. and Peptostreptococcus spp. Pasteurella spp., and Globicatella spp. taxa have been found in healthy suckling piglets, which suggests that $C D$ and other potential pathogens could contribute to a gut inflammation and AR spread [16]. It is known that certain groups of bacteria such as Proteobacteria and enterococci carry a high abundance of AR genes [24]. Under normal conditions, their 
cell concentration is not high enough for efficient AR gene transfer. However, in a state of dysbiosis where colonization resistance is disrupted, an expansion of Proteobacteria and enterococci may lead to an increased abundance of AR genes, as we have found in artificially reared piglets. Moreover, it has been shown that gut inflammation boosts AR gene transfer between pathogenic and commensal Enterobacteriaceae in mice [6]. Similarly, non-virulent CD may acquire virulence from virulent $C D$ types via conjugative transposons which are known to encode toxin and AR genes, therefore increasing the AR load in the gut environment $[25,26]$.

In pigs, for example, reports have linked the use of supra-nutritional levels of trace elements such as $\mathrm{Cu}$ and $\mathrm{Zn}$ in swine diets with an increased prevalence of multi-resistant E. colistrains [27]. A co-selection for AR genes within the intestinal microbial communities during or after an initial state of dysbiosis could even further promote an expansion of the bacterial resistome.

In the present study, a total of 83 AR genes were identified, conferring resistance to members of 11 classes of antimicrobial agents. A higher abundance of these genes was determined in FP as well as FP$C D$ piglets as compared to the other groups. However, the abundance of certain AR genes such as tet( $Q)$, tet(W), Inu(C), Isa(E), or ant(6)-Ib in S, SP and WP groups was apparently linked to Megasphaera, Bifidobacterium, Lactobacillus, or Prevotella thereby confirming these genera as common carriers of these AR genes [28]. Moreover, the observed co-occurrence of some AR genes among all groups could be attributed to microbial community composition and co-occurrence of certain bacterial taxa in general but also to gene co-localization in bacterial genomes. Plasmid co-location of AR genes such as bla $C T X-M$ genes and $m p h(\mathrm{~A})$ was previously reported in extended-spectrum $\beta$-lactamase-producing $E$. coli isolates from farm animals (including pigs) in Germany [29].A recent study showed clusters of co-occurring AR

genes in pig environments including such as $\operatorname{tet}(\mathrm{W}), \operatorname{tet}(\mathrm{Q}), \operatorname{tet}(\mathrm{B}), \operatorname{mef} A, b / a_{\text {TEM }}[30]$. The present study shows that such co-occurrences are rather dynamic and that the abundance of AR genes largely depends on the microbiome composition. As shown, the latter is influenced by age (e.g. SP vs. WP vs. S), diet and rearing environment (e.g. SP vs. FP), and CDI (FP vs. FP-CD). However, our study also supports the hypothesis that metagenomic approaches may help to identify and monitor antibiotic resistance patterns in gut microbial communities in the pig [31].

\section{Conclusion}

Taken together, we show that artificial rearing and formula feeding alone or combined with CDI and an immature-like microbiome promote a highly abundant bacterial resistome in neonatal piglets. Following the "One Health" concept, the relevance for therapeutic resistance or the role of formula-fed neonatal pigs as potential AR gene shedders later in life needs to be further elucidated.

\section{Materials And Methods}

\section{Animals, experimental setup and sampling}


The aim of the present study was to determine a possible link between the intestinal microbiome development and the resistome in neonatal piglets fed formula and infected with $C D$, as compared to their mothers, suckling littermates, and weaned littermates later in life.

Purebred sows ('German Landrace') and their suckling and weaned piglets were used in the study. Sows received a standard lactation diet and were not treated with antibiotics before or during the experiments. During the suckling period, sow milk was the only nutrient source and piglets had no access to creep feed. Immediately after birth, a total of six piglets from three sows (two piglets per sow) were randomly selected and transferred into artificial rearing units $[12,17]$. Three piglets (one from each sow) were fed formula (formula piglets, FP), whereas three animals were fed formula and infected at $6 \mathrm{~h}$ after birth with $10^{8} \mathrm{CFU}$ Clostridium difficile ribotype 078 (FP-CD) spores. At 28 days of age, the remaining suckling piglets were weaned and fed a standard cereal-soy-based weaning diet without antibiotics or special feed additives (e.g. organic acids or probiotics). All diets met the nutrient requirements according to current recommendations. For microbiome analysis, fecal samples were taken from four sows (S) and a pooled sample from their suckling piglets $(n=4)$ at day 5 (suckling piglets; SP) and from two piglets per litter $(m / f)$ two weeks after the weaning at day $42(n=8$ weaned piglets, WP). Fecal samples from FP $(n=3)$ and FP-CD $(\mathrm{n}=3)$ animals were taken on day 5 .

\section{DNA extraction and sequencing}

Total genomic DNA was extracted using the QIAamp Fast DNA Stool Mini Kit (Qiagen, Hilden, Germany) with bead-beating to improve DNA recovery from spore-forming bacteria [32]. After DNA quality control using the Genomic DNA Analysis ScreenTape on a 2200 TapeStation Instrument (Agilent Technologies) and concentration measurement using the Qubit dsDNA BR Assay Kit (Thermo Fisher Scientific), shotgun metagenomic libraries were generated using the Nextera DNA Library Preparation Kit (Illumina) according to the manufacturers' instructions. Libraries were quality-controlled using the D5000 DNA Analysis ScreenTape on a 2200 TapeStation Instrument (Agilent Technologies), and sequenced on an Illumina NextSeq500 with $2 \times 150$ bp. Finally, sequencing reads were demultiplexed based on the used Nextera indices (dual indexing principle). The average number of obtained sequences was $15942818 \pm 4775$ 046 reads per sample.

\section{Data analyses}

The FLEXBAR tool was used to remove adapter sequences from the metagenomic reads and discard reads with length shorter than 100 nucleotides [33]. We mapped quality checked sequences (89\% of the original sequencing reads) against reference genomes of 13193 different bacterial and archaeal species taken from NCBI (ftp://ftp.ncbi.nlm.nih.gov/genomes/genbank/). We used the yara ("yet another read aligner") tool to perform read mapping [34]. The resulting mapping files were further analyzed using the Species Level Identification of Microorganisms from Metagenomes (SLIMM) tool to produce for taxonomic profiles of each sample [35]. We calculated relative abundances of individual taxa using the SLIMM tool. SLIMM defines relative abundance as the number of reads assigned uniquely to a taxon divided by the total number of mapped reads then corrected for genome sequence length, thus allowing 
for downstream analysis of taxa diversity and abundance. We used a relative abundance cut-off of $0.01 \%$ to consider a given taxon of bacteria present.

For resistome analysis, we mapped metagenomic reads against version 1.1.2 of the Comprehensive Antibiotic Resistance Database (CARD; http://arpcard.mcmaster.ca) using yara read mapper. Then we used the number of reads mapped to each resistance gene per $1000 \mathrm{bp}$ as an indicator for the relative sequence abundance for each gene. Only genes coding for a true resistance were used for further analysis, whereas genes related to multidrug resistance, transcription factors or regulating factors were deleted from the initial list. Within AR genes coding for $\beta$-lactam resistance, 'bla $a_{C T X}-M^{\text {type' }}$ and 'bla like' genes were summarized as one group, whereas the genes related to vancomycin (glycopeptide) resistance were summarized under the term 'vanB gene cluster'. Using the relative abundance of AR genes (normalized by total metagenomic sequences per sample), a heatmap was calculated using the 'heatmap2' package in R. The hierarchical clustering was performed using the ward.D method. To analyze the interactions between intestinal microbial genera (explanatory variables) with the abundance of antimicrobial resistance genes (response variables) in sows and their differently reared piglets, a multivariate analysis was performed using the CANOCO statistical package. Explanatory and response data for each animal were used for a constrained linear ordination analysis (redundancy analysis, RDA). Significance of the overall ordination model as well as the effect of explanatory variables during development of the ordination model was tested using Monte Carlo permutation test $(n=499)$.

\section{Abbreviations}

AR-Antimicrobial resistance; CARD - Comprehensive Antibiotic Resistance Database; CD-Clostridium difficile, $\mathrm{CDI}$-Clostridium difficile infection; FP-Formula-fed; FP-CD-Formula-fed, Clostridium difficileinfected; SLIMM - Species Level Identification of Microorganisms from Metagenomes; RDA-Redundancy Analysis; S-Sows; SP-Suckling piglets; WP-Weaned piglets

\section{Declarations}

\section{Ethical approval}

All experimental procedures involving artificial rearing and $C D$ infection of piglets were approved by the local authorities (Landesamt für Gesundheit und Soziales, Reg \# G0255/14).

\section{Consent for publication}

Not applicable.

\section{Availability of data and materials}


The metagenomic sequences generated during and analyzed during the current study have been deposited in the European Nucleotide Archive (ENA) under accession code PRJEB19642 (https://www.ebi.ac.uk/metagenomics/projects/ERP021695).

\section{Competing interests}

The authors declare that they have no competing interests.

\section{Funding}

This work was funded through the Research Committee at the Freie Universität Berlin, the German Research Foundation (Pi 946/2-1), the SporeBiotic research project within the Animal Health and Welfare ERA-Net (ANIHWA, 031A357), and the Cluster of Excellence "Inflammation at Interfaces". T.H.D was a member of a PhD program at the International Max Planck Research School for Computational Biology and Scientific Computing.

\section{Authors contributions}

R.P., Ł.G., W.V., B.S. and J.Z. designed the study. R.P. and Ł.G. conducted the animal experiments. F.-A.H. and A.F. performed metagenomics sequencing. T.H.D. and K.R.; curated and analysed the raw sequencing data, R.P., T.H.D., L.P., S.S. and W.V. performed in-depth data analysis and statistics. data curation, R.P., T.H.D., Ł.G., B.S. and S.S. drafted and edited the manuscript. R.P., T.H.D., and L.P. visualised the data. All authors read and approved the final manuscript.

\section{Acknowledgements}

We would like to thank the laboratory and animal care staff of the Institute of Animal Nutrition, Freie Universität Berlin, for technical support.

\section{References}

1. Bush K, Courvalin P, Dantas G, Davies J, Eisenstein B, Huovinen P, Jacoby GA, Kishony R, Kreiswirth BN, Kutter E, Lerner SA, Levy S, Lewis K, Lomovskaya O, Miller JH, Mobashery S, Piddock LJ, Projan S, Thomas CM, Tomasz A, Tulkens PM, Walsh TR, Watson JD, Witkowski J, Witte W, Wright G, Yeh P, Zgurskaya HI. Tackling antibiotic resistance. Nat Rev Microbiol. 2011;9:894-6.

2. Carlet $\mathrm{J}$. The gut is the epicentre of antibiotic resistance. Antimicrob Resist Infect Control. 2012;1:39. 
3. van Schaik W. The human gut resistome. Philos Trans R Soc Lond B Biol Sci. 2015;370:20140087.

4. Joyce A, McCarthy CGP, Walsh M. F. Antibiotic resistomes of healthy pig faecal metagenomes. Microb Genom. 2019;5:e000272.

5. Xiao L, Estellé J, Kiilerich P, Ramayo-Caldas Y, Xia Z, Feng Q, Liang S, Pedersen A, Kjeldsen NJ, Liu C, Maguin E, Doré J, Pons N, Le Chatelier E, Prifti E, Li J, Jia H, Liu X, Xu X, Ehrlich SD, Madsen L, Kristiansen K, Rogel-Gaillard C, Wang J. A reference gene catalogue of the pig gut microbiome. Nat Microbiol. 2016; 16161.

6. Stecher B, Denzler R, Maier L, Bernet F, Sanders MJ, Pickard DJ, Barthel M, Westendorf AM, Krogfelt KA, Walker AW, Ackermann M, Dobrindt U, Thomson NR, Hardt WD. Gut inflammation can boost horizontal gene transfer between pathogenic and commensal Enterobacteriaceae. Proc Natl Acad Sci U S A. 2012;109:1269-74.

7. Spigaglia P, Barbanti F, Mastrantonio P, Ackermann G, Balmelli C, Barbut F, Bouza E, Brazier J, Delmée M, Drudy D, Kuijper E, Ladas H, Mastrantonio P, Nagy E, Pituch H, Poxton I, Rupnik M, Wullt W, Yücesoy M. Multidrug resistance in European Clostridium difficile clinical isolates. J Antimicrob Chemother. 2011;66:2227-34.

8. Millan B, Park H, Hotte N, Mathieu O, Burguiere P, Tompkins TA, Kao D, Madsen KL. Fecal microbial transplants reduce antibiotic-resistant genes in patients with recurrent Clostridium difficile infection. Clin Infect Dis. 2016;62:1479-86.

9. Moono P, Foster NF, Hampson DJ, Knight DR, Bloomfield LE, Riley TV. Clostridium difficile infection in production animals and avian species: A review. Foodborne Pathog Dis. 2016;13:647-55.

10. Knetsch CW, Kumar N, Forster SC, Connor TR, Browne HP, Harmanus C, Sanders IM, Harris SR, Turner L, Morris T, Perry M, Miyajima F, Roberts P, Pirmohamed M, Songer JG, Weese JS, Indra A, Corver J, Rupnik M, Wren BW, Riley TV, Kuijper EJ, Lawley TD. Zoonotic Transfer of Clostridium difficile Harboring Antimicrobial Resistance between Farm Animals and Humans. J Clin Microbiol. 2018; pii:e01384-17.

11. Debast SB, Van Leengoed LAMG, Goorhuis A, Harmanus C, Kuijper EJ, Bergwerff AA. Clostridium difficile PCR ribotype 078 toxinotype $\mathrm{V}$ found in diarrhoeal pigs identical to isolates from affected humans. Environ Microbiol. 2009;11:505-11.

12. Grześkowiak Ł, Martínez-Vallespín B, Dadi TH, Radloff J, Amasheh S, Heinsen FA, Franke A, Reinert K, Vahjen W, Zentek J, Pieper R. Formula-feeding predisposes neonatal piglets to Clostridium difficile gut infection. J Infect Dis. 2018;217:1442-52.

13. Bian G, Ma S, Zhu Z, Su Y, Zoetendal EG, Mackie R, Liu J, Mu C, Huang R, Smidt H, Zhu W. Age, introduction of solid feed and weaning are more important determinants of gut bacterial succession in piglets than breed and nursing mother as revealed by a reciprocal cross-fostering model. Environ Microbiol. 2016;18:1566-77.

14. Mach N, Berri M, Estellé J, Levenez F, Lemonnier G, Denis C, Leplat JJ, Chevaleyre C, Billon Y, Doré J, Rogel-Gaillard C, Lepage P. Early-life establishment of the swine gut microbiome and impact on host phenotypes. Environ Microbiol Rep. 2015;7:554-69. 
15. Koenig JE, Spor A, Scalfone N, Fricker AD, Stombaugh J, Knight R, Angenent LT, Ley RE. Succession of microbial consortia in the developing infant gut microbiome. Proc Natl Acad Sci U S A. 2011;108(Suppl 1):4578-85.

16. Grześkowiak $Ł$, Dadi TH, Zentek J, Vahjen W. Developing Gut Microbiota Exerts Colonisation Resistance to Clostridium (syn. Clostridioides) difficile in Piglets. Microorganisms. 2019;7:pii:E218.

17. Pieper R, Vahjen W, Zentek J. Intestinal lactose and mineral concentration affect the microbial ecophysiology along the gastrointestinal tract of formula fed neonatal piglets. J Anim Sci. 2016;94:3786-95.

18. Penders J, Vink C, Driessen C, London N, Thijs C, Stobberingh EE. Quantification of Bifidobacterium spp., Escherichia coli and Clostridium difficile in faecal samples of breast-fed and formula-fed infants by real-time PCR. FEMS Microbiol Lett. 2005;243:141-7.

19. Rahman SF, OIm MR, Morowitz MJ, Banfield JF. Machine learning leveraging genomes from metagenomes identifies influential antibiotic resistance genes in the infant gut microbiome. mSystems. 2018;3:e00123-17.

20. Grześkowiak $Ł$, Pieper R, Hong HA, Cutting SM, Vahjen W, Zentek J. Impact of early-life events on the susceptibility to Clostridium difficile colonisation and infection in the offspring of the pig. Gut Microbes. 2019;10:251-9.

21. Grześkowiak $Ł$, Pieper R, Kröger S, Martínez-Vallespín B, Hauser A, Niesner R, Vahjen W, Zentek J. Porcine milk protects the IPEC-J2 cells and piglet colon epithelium against Clostridioides (syn. Clostridium) difficile toxin-induced effects. Microorganisms. 2020;8:142.

22. Buffie CG, Bucci V, Stein RR, McKenney PT, Ling L, Gobourne A, No D, Liu H, Kinnebrew M, Viale A, Littmann E, van den Brink MR, Jenq RR, Taur Y, Sander C, Cross JR, Toussaint NC, Xavier JB, Pamer EG. Precision microbiome reconstitution restores bile acid mediated resistance to Clostridium difficile. Nature. 2015;517:205-8.

23. Kim YG, Sakamoto K, Seo SU, Pickard JM, Gillilland MG 3rd, Pudlo NA, Hoostal M, Li X, Wang TD, Feehley T, Stefka AT, Schmidt TM, Martens EC, Fukuda S, Inohara N, Nagler CR, Núñez G. Neonatal acquisition of Clostridia species protects against colonization by bacterial pathogens. Science. 2017;356:315-9.

24. Liu B, Pop M. ARDB-Antibiotic Resistance Genes Database. Nucleic Acids Res. 2009;37:D443-7.

25. Brouwer MSM, Roberts AP, Hussain H, Williams RJ, Allan E, Mullany P. Horizontal gene transfer converts non-toxigenic Clostridium difficile strains into toxin producers. Nat Commun. 2013;4:2601.

26. Wasels F, Monot M, Spigaglia P, Barbanti F, Ma L, Bouchier C, Dupuy B, Mastrantonio P. Inter- and Intraspecies Transfer of a Clostridium difficile Conjugative Transposon Conferring Resistance to MLS $_{\mathrm{B}}$. Microb Drug Resist. 2014;20:555-60.

27. Bednorz C, Oelgeschläger K, Kinnemann B, Hartmann S, Neumann K, Pieper R, Bethe A, Semmler T, Tedin K, Schierack P, Wieler LH, Guenther S. The broader context of antibiotic resistance: zinc feed supplementation of piglets increases the proportion of multi-resistant Escherichia coli in vivo. Int $\mathrm{J}$ Med Microbiol. 2013;303:396-403. 
28. Roberts MC, Schwarz S. Tetracycline and phenicol resistance genes and mechanisms: Importance for agriculture, the environment, and humans. J Environ Qual. 2016;45:576-92.

29. Schink A-K, Kadlec K, Kaspar H, Mankertz J, Schwarz S. Analysis of extended-spectrum- $\beta$-lactamaseproducing Escherichia coli isolates collected in the GERM-Vet monitoring programme. J Antimicrob Chemother. 2013;68:1741-9.

30. Johnson TA, Stedtfeld RD, Wang Q, Cole JR, Hashsham SA, Looft T, Zhu YG, Tiedje JM. Clusters of antibiotic resistance genes enriched together stay together in swine agriculture. mBio. 2016;7:e02214-15.

31. Munk P, Andersen VD, de Knegt L, Jensen MS, Knudsen BE, Lukjancenko O, Mordhorst H, Clasen J, Agers $\varnothing$ Y, Folkesson A, Pamp SJ, Vigre H, Aarestrup FM. A sampling and metagenomic sequencingbased methodology for monitoring antimicrobial resistance in swine herds. $J$ Antimicrob Chemother. 2017;72:385-92.

32. Grześkowiak $\measuredangle$, Zentek J, Vahjen W. Determination of the extent of Clostridium difficile colonisation and toxin accumulation in sows and neonatal piglets. Anaerobe. 2016;40:5-9.

33. Dodt M, Roehr JT, Ahmed R, Dieterich C. FLEXBAR-Flexible Barcode and Adapter Processing for Next-Generation Sequencing Platforms. Biology. 2012;1:895-905.

34. Siragusa E, Weese D, Reinert K. Fast and accurate read mapping with approximate seeds and multiple backtracking. Nucleic Acids Res. 2013;41:e78.

35. Dadi TH, Renard BY, Wieler LH, Semmler T, Reinert K. SLIMM: species level identification of microorganisms from metagenomes. PeerJ. 2017;5:e3138.

\section{Figures}


A

(5 days after farrowing)

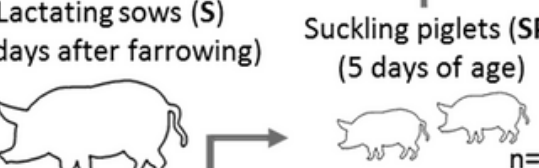

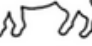

$n=4$

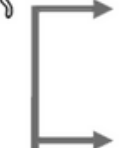

Weaned piglets (WP)

(42 days of age)

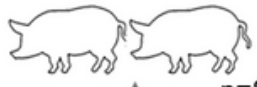

$n=8$

uckling piglets (SP)

days of age)

जoros wors $n=4$

Formula-fed (FP)

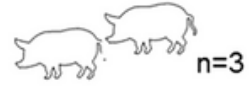

Formula+CD (FP-CD)

B

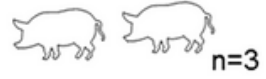

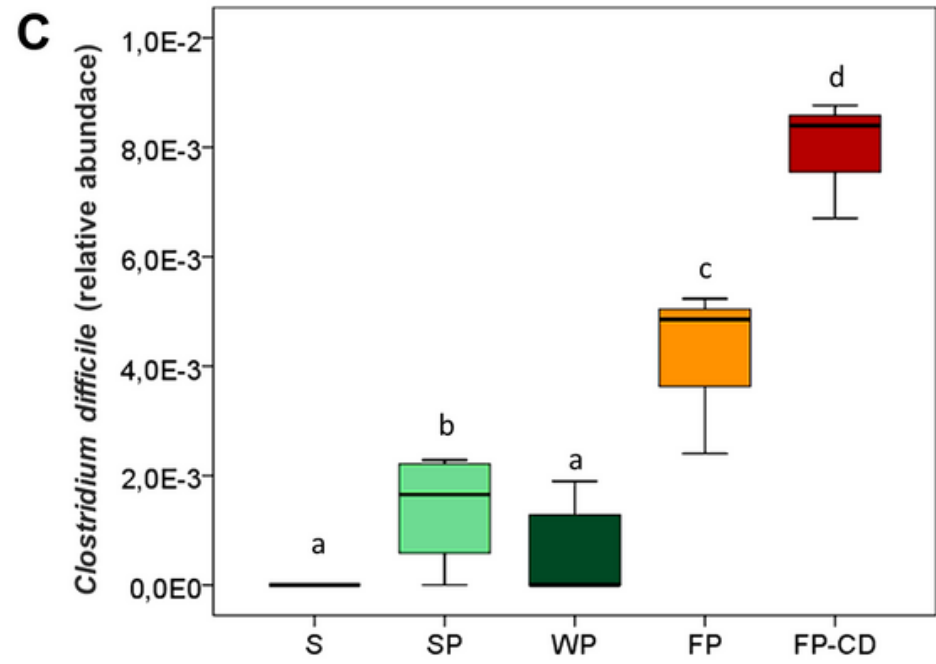

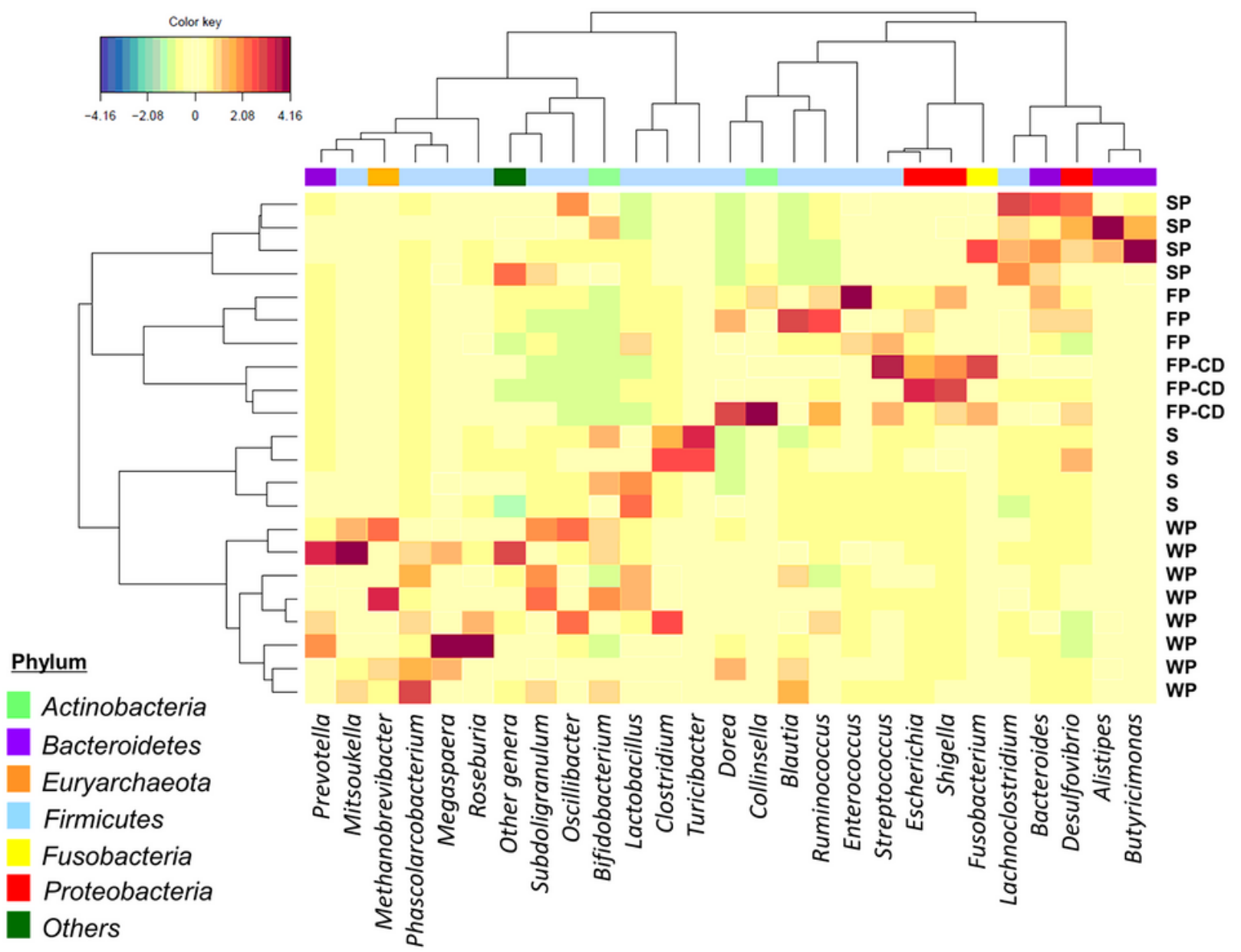

Figure 1

A-C. Influence of early-life environment, diet and Clostridium difficile (R078) infection on intestinal microbiome composition in sows and their offspring. (A) Experimental setup indicating the origin of fecal samples from sows (S), their suckling piglets (SP), weaned piglets (WP) from the same litters, artificially reared and formula-fed littermates (FP), and artificially reared and formula-fed littermates infected with Clostridium difficile (FP-CD) 6h after birth, (B) heatmap showing the relative abundance of the most 
prevalent identified bacterial genera, and (C) relative abundance of sequences mapping to Clostridium difficile in metagenomic samples. Different superscripts $(a b c d)$ denote significant $(P<0.05)$ differences.
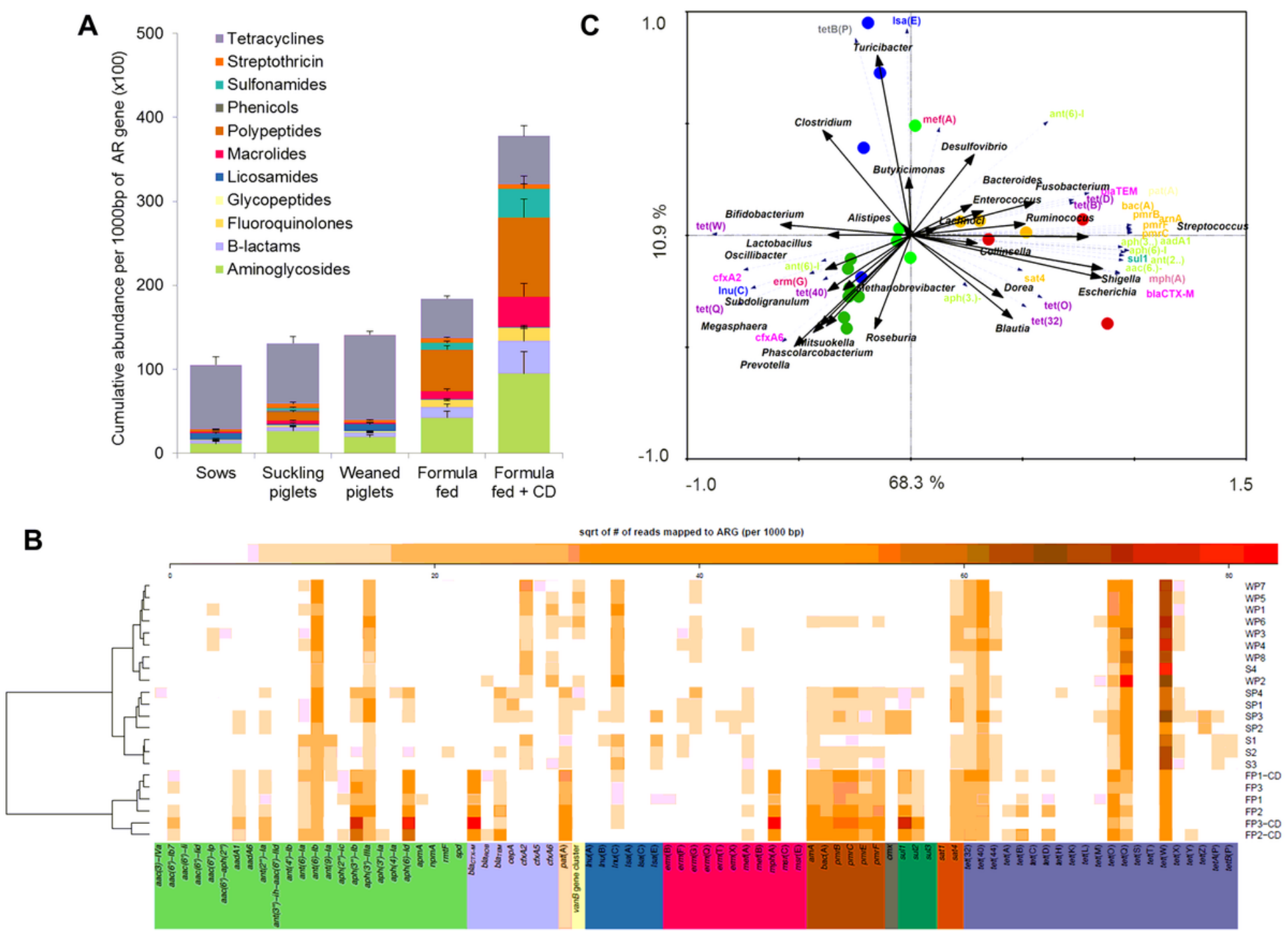

\section{Figure 2}

A-C. Resistome in sows and their offspring as determined by metagenomic sequencing. (A) Cumulative abundance of sequences (given as \# of sequences per $1000 \mathrm{bp}$ of resistance gene) related to AR genes assigned to 11 antibiotic classes in sows (S), their suckling (SP) and weaned piglets (WP), formula-fed littermates (FP), or formula-fed littermates infected with Clostridium difficile (FP-CD). (B) heatmap showing 83 individual AR genes that were present in individual sows (S1-4), their suckling (SP1-4) and weaned piglets (WP1-8), formula-fed littermates (FP1-3), or formula-fed littermates infected with Clostridium difficile (FP-CD1-3). (C) RDA triplot of the most predominant bacterial genera (solid black arrows) in relation to the 35 most abundant AR genes (dashed grey arrows) in sows (blue circles), their suckling (light green circles) and weaned piglets (dark green circles), formula-fed littermates (orange circles), or formula-fed littermates infected with Clostridium difficile (red circles). The length, direction and the angle between arrows are an approximation of correlations between variables or variables and canonical axes (e.g. $\left.a=0 \% / r=1 ; a=90^{\circ} / r=0 ; a=180^{\circ} / r=-1\right)$. Percentage values on axis 1 and 2 indicate 
the variability described through the canonical axis. Color codes indicate resistance gene families according to Figure 2A. 